\title{
Performance Investigation of Dual Wavelength Fiber Laser using different SOAs
}

\author{
Y. S. Ong' , A. H. Sulaiman' ${ }^{2}$ F. Abdullah', K. Y. Lau ${ }^{3}$, N. Md. Yusoff $\mathbf{1}^{*}$ \\ ${ }^{1}$ Razak Faculty of Technology and Informatics, \\ Universiti Teknologi Malaysia Kuala Lumpur, Jalan Sultan Yahya Petra, 54100 Kuala Lumpur, MALAYSIA \\ ${ }^{2}$ Institute of Power Engineering, \\ Universiti Tenaga National, Jalan IKRAM-UNITEN, 43000 Kajang, Selangor, MALAYSIA \\ ${ }^{3}$ Department of Electronics and Nanoengineering, \\ Aalto University, Tietotie 3, 02150 Espoo, FINLAND \\ *Corresponding Author
}

DOI: https://doi.org/10.30880/ijie.2020.12.06.030

Received 8 April 2020; Accepted 3 August 2020; Available online 30 August 2020

\begin{abstract}
We investigated the performance of a dual-wavelength fiber laser using different types of semiconductor optical amplifiers (SOAs) utilizing fiber Bragg gratings (FBGs) as comb filter. The lasing performances of linear SOA (LSOA), nonlinear SOA (NLSOA) and booster optical amplifier (BOA) at different current setting were observed. LSOA produced the flattest spectrum based on its peak power difference at only $4.5 \mathrm{~dB}$ as compared to $66.4 \mathrm{~dB}$ using BOA. BOA has the worst flatness because its polarization sensitivity is the highest as compared to LSOA and NLSOA. Overall, the best lasing performance is when LSOA is used due to the lowest peak power difference with high peak power at $-17.6 \mathrm{dBm}$.
\end{abstract}

Keywords: Dual wavelength fiber laser; semiconductor optical amplifier; fiber Bragg grating

\section{Introduction}

Multiwavelength fiber laser (MWFL) has been a major attraction to researchers and industry due to its potential application in optical communications, dense wavelength division multiplexing system and optical fiber sensor. Many researchers have demonstrated MWFL based on several comb filters such as Lyot filter [1,2], Sagnac loop mirror [3], Mach Zehnder interferometer [4] and FBGs [5]. Among the comb filters, FBGs are more stable because it not depends on polarization state to achieve flat lasing lines. FBGs have been utilized to demonstrate stable triple-wavelength [6], a fiber sensor that capable of simultaneously measuring temperature and strain [7], switchable MWFL with tunable wavelength spacing [8] and high stability half-open cavity multiwavelength random erbium-doped fiber laser [9].

The main benefit of using SOA in MWFL is that it has the property of inhomogeneous gain broadening to alleviate the issue of mode competition that is commonly present in the generation of multiple lasing wavelengths at room temperature [10-13]. Low mode competition in SOA is a significant advantage over erbium-doped fiber amplifiers (EDFAs), where the number of laser modes is generally limited because of high mode competition resulting from the homogenous gain broadening of erbium ions. Other advantages are compactness, low cost, lower power consumption as well as mass-producible for the application of fiber laser and amplifier. The operation of SOA is more straightforward because the photons are generated through the use of an electrical pumping scheme, which simplifies the amplifier design. 
In our work, we used FBGs for the generation of dual-wavelength fiber laser (DWFL). Several techniques for generating DWFL have been implemented either using a section of polarization-maintaining (PM) fiber [14], 3-stage all-fiber Lyot filter [15], two written FBGs in a photosensitive and PM erbium-doped fiber [16], a multi-segmented allPM fiber ring laser [17], two Sagnac loop mirrors [18] and two FBGs [19]. However, from the best of our knowledge, their works did not investigate DWFL at a different type of SOAs, which was never being reported previously. In this work, we investigate the performance of DWFL based on different types of SOA in LSOA, NLSOA and BOA. LSOA has presented the flattest dual-wavelength with a peak power difference of $4.5 \mathrm{~dB}$, followed by NLSOA at $6.2 \mathrm{~dB}$. BOA has the highest polarization sensitivity that leads to the worst flatness of DWFL.

\section{Experiment Setup}

Fig. 1 depicts the experimental setup for the DWFL based on three different types of SOA which manufactured by Thorlabs, that are LSOA (SOA1013S), NLSOA (SOA1117S) and BOA (BOA1004S), driven by a laser diode controller (LDC) from ILX Lightwave, model LDC-3900. Their amplified spontaneous emission (ASE) sources are filtered out by two FBGs at a center wavelength of $1545 \mathrm{~nm}$ and $1555 \mathrm{~nm}$. An isolator is used to ensure the cavity oscillation is in a counterclockwise direction. A circulator is used to allow two light directions only which are from Port 1 to Port 2 and Port 2 to Port 3. Thus, the light from SOA unable to travel from Port 3 to Port 2 and Port 3 to Port 1. The light from Port 1 cannot travel to Port 3, as it must go to Port 2 first. The laser output is tapped out using $10 \%$ of tap coupler to an optical spectrum analyzer (OSA), with the majority $90 \%$ of the light is going back to the internal cavity circulation for measurement. The resolution and sensitivity of the OSA are set to $0.02 \mathrm{~nm}$ and high1, respectively.

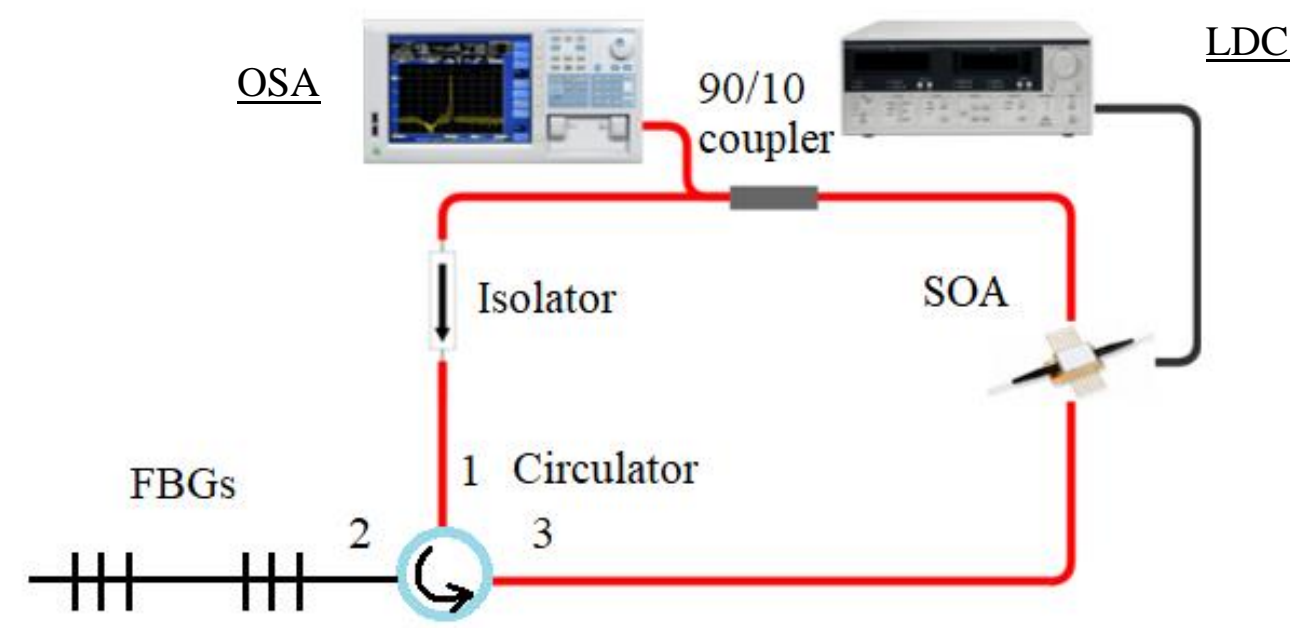

Fig. 1 - The experimental setup for generating the DWFL

\section{Structure and Material of SOA}

The structure of SOA is designed in a hermetic butterfly package. Typically, the chip size of an SOA is approximately $1.2 \mathrm{~mm}$. The LSOA, as pictured in Fig. 2(a), is finely cased in a rectangular shape package for secure handling and orderly design. In the casing, the fiber pigtails are looped and fastened tidily. NLSOA and BOA are also cased similarly to LSOA with larger loop diameter to reduce bending loss. The material of the casing is made from a particular material for current grounding.

The SOA architecture is illustrated in Fig. 2(b). The figure shows the flow of the injected current, the amplified output signal, the SOA facets and the active layer of the SOA. The width of the active layer is typically $\sim 0.6$ to $2.0 \mathrm{~mm}$ and is integral for the amplification process. The active layer is located between the anti-reflection coatings applied to the input and output facets. The active layer region produces high insertion loss at approximately $5 \mathrm{~dB}$ due to the use of non-glass material. The input and the output facets allow light to pass from the optical fiber into the active layer for the amplification process. Another essential component is coupling optics used to couple light to the fiber pigtails and into the active region. The semiconductor material is designed in such a way so that the optical signal is confined only in the active region to increase efficiency as well as reduce optical losses. 


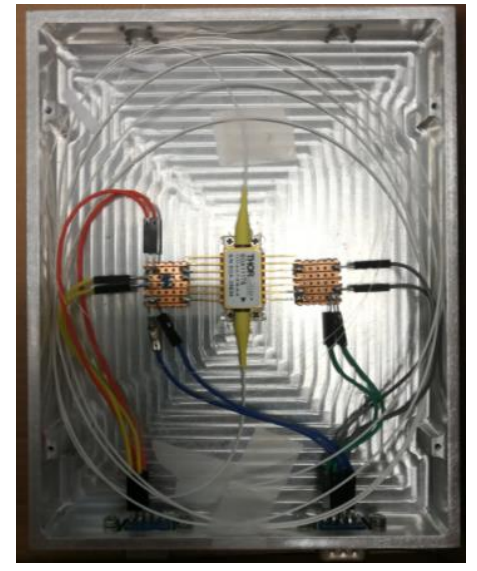

(a)

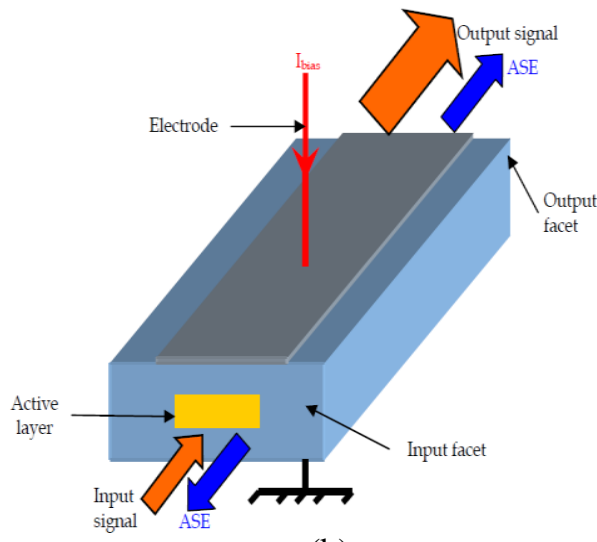

(b)

Fig. 2.4 - (a) The aerial view of actual SOAs that is used in the lab; (b) SOA's architecture and other signage [20]

Further information on nonlinearity, category and usage of the SOAs is shown in Table 1. LSOA is an inline SOA and has low nonlinearity, while NLSOA and BOA have high nonlinearity. BOA is a booster type and usually located at the beginning of an optical communication system to boost the optical input power. For SOAs under an inline category, their function is to amplify the optical signal for every $80 \mathrm{~km}$ to $100 \mathrm{~km}$. In a fiber laser, SOAs were usually used as the ASE source to produce a single, dual or multiple laser wavelengths at selected wavelengths that depend on the use of comb filter.

Table 1 - The nonlinearity, category and usage of the SOAs

\begin{tabular}{ccccc}
\hline $\begin{array}{c}\text { SOA type and } \\
\text { model number }\end{array}$ & Nonlinearity & Category & $\begin{array}{c}\text { Maximum } \\
\text { signal gain }\end{array}$ & Usage in transmission link \\
\hline LSOA (SOA1013S) & Low & Inline & $\underline{13}$ & To amplify optical signal every 80 to 100 km \\
\hline NLSOA (SOA1117S) & High & Inline & $\underline{20}$ & To amplify optical signal every 80 to 100 km \\
\hline BOA (BOA1004S) & High & Booster & $\underline{27}$ & To boost input power \\
\hline
\end{tabular}

\section{Results and Discussions}

Fig. 3 depicts the ASEs of LSOA, NLSOA and BOA spanning from $1400 \mathrm{~nm}$ to $1650 \mathrm{~nm}$. The spectra were recorded at their typical bias current at $500 \mathrm{~mA}$ for LSOA and NLSOA and $600 \mathrm{~mA}$ for BOA. Even though the maximum current setting is at $750 \mathrm{~mA}$, we only set the highest SOA setting to a typical setting to avoid SOA damage. The peak power of ASE for LSOA, NLSOA and BOA is $-32 \mathrm{dBm},-38 \mathrm{dBm}$ and $-35 \mathrm{dBm}$, respectively. Spectrum width is the ASE bandwidth within $10 \mathrm{~dB}$, which measured at $120 \mathrm{~nm}, 110$ and $170 \mathrm{~nm}$ for LSOA, NLSOA and BOA, respectively. BOA has the widest ASE, followed by LSOA and NLSOA. The spectrum bandwidth is having a distinct spectral shape as they follow values of saturated output power (SOP) of each SOA. The BOA has the highest SOP, which had led to the broadest spectrum bandwidth. 


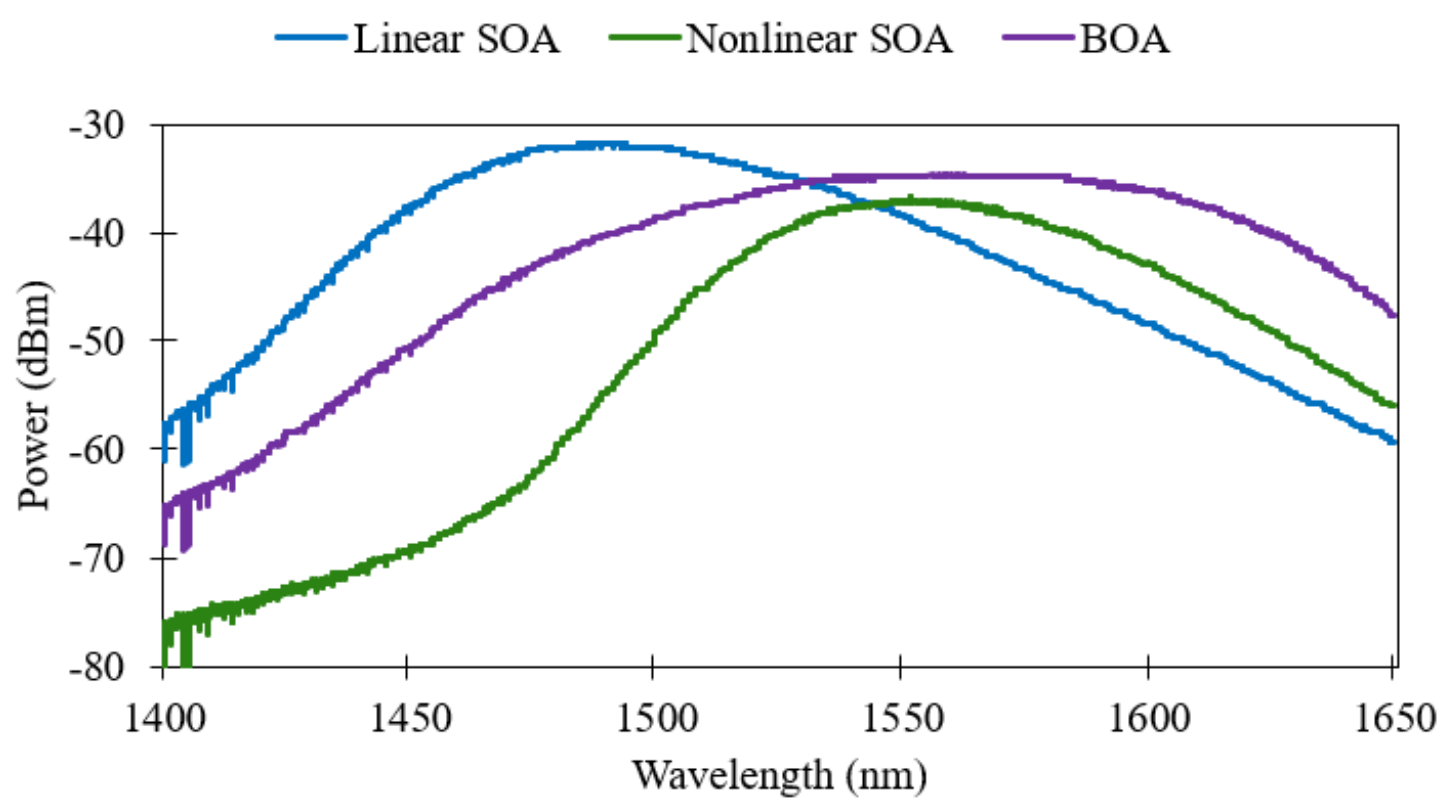

Fig. 3 - The ASE of LSOA, NLSOA and BOA at their maximum current setting

FBG is a type of distributed Bragg reflector constructed in a short segment of optical fiber that reflects particular wavelengths of light and transmits all others. In this experiment, the FBGs were used as an inline optical filter to block specific wavelengths, or as a wavelength-specific reflector. Fig. 4(a) depicts the configuration setup to check the FBG's reflectivity. This characterization was done without connecting isolator to the FBGs that cause to the existence of ASE noise due to back reflection of the light at the end of FBG. The FBG used has reflectivity of $97 \%$ with a center wavelength of $1545 \mathrm{~nm}$ and $1555 \mathrm{~nm}$. As depicted in Fig. 4(b), the peak-to-floor for a center wavelength of $1545 \mathrm{~nm}$ and $1555 \mathrm{~nm}$ is $6.4 \mathrm{~dB}$ and $6 \mathrm{~dB}$, respectively. These FBGs have the function to filter out the ASE and allow only selected wavelength equivalent to their center wavelength. The two peaks oscillate in the lasing cavity to produce dualwavelength output. Fig. 4(c) depicts the configuration setup to check the FBG's transmissivity. Fig. 4(d) illustrates the transmission spectrum of the FBGs with two different wavelength valleys, which are occurred at $1545 \mathrm{~nm}$ and 1555 $\mathrm{nm}$. The FBG has a transmissivity of $97 \%$ with a center wavelength of $1545 \mathrm{~nm}$ and $1555 \mathrm{~nm}$. For a center wavelength of $1545 \mathrm{~nm}$ and $1555 \mathrm{~nm}$, the peak-to-floor was measured at $9 \mathrm{~dB}$ and $10 \mathrm{~dB}$, respectively.

Fig. 5(a-c) depict the output spectra of the dual laser wavelength using different SOAs. The peak wavelengths of LSOA, NLSOA and BOA were indicated in the figure. The dual laser wavelength based on LSOA and NLSOA shows almost equal power between the two-lasing channel. However, NLSOA produces much lower output power than LSOA by about $50 \mathrm{~dB}$. The high loss can be rectified by adjusting the polarization state in the cavity using a polarization controller (PC). The peak power difference will be discussed further in Table 2. For the DWFL setup using BOA, the dual-channel output is not flat as the $1555 \mathrm{~nm}$ peak get all the gain. The peak difference of about $65 \mathrm{~dB}$ makes the 1545 $\mathrm{nm}$ peak more suitable to be considered as side mode rather than a lasing channel. The flatness issue can be solved by adding a PC in the setup to optimize the lasing lines because BOA has high sensitivity. LSOA has the best lasing performance since its peak power difference is the lowest with high peak power at $-17.6 \mathrm{dBm}$.

Fig. 6 (a-c) show peak power increment of the DWFL due to the increase of SOAs' bias current. The two peak powers are Peak $1(1545 \mathrm{~nm})$ and Peak $2(1555 \mathrm{~nm})$ are observed at $-80 \mathrm{dBm}$ for all SOAs when the bias current is set to $50 \mathrm{~mA}$. Fig. 6(a) shows the peak power of DWFL based on LSOA, as when the LSOA current is set within $150 \mathrm{~mA}$ to $200 \mathrm{~mA}$, both peak powers have increased to approximately $-20 \mathrm{dBm}$. With a further increment of SOA current until $500 \mathrm{~mA}$, Peak 1 and Peak 2 is raised slightly to approximately $-15 \mathrm{dBm}$. The peak power of NLSOA-based DWFL is shown in Fig. 6(b), as it possessed at least $40 \mathrm{~dB}$ gain difference starting from $250 \mathrm{~mA}$. This 40 -dB gain difference is due to the high polarization sensitivity imposed by the NLSOA. Fig. 6(c) shows the peak powers of DWFL using BOA, with a large peak difference at approximately $70 \mathrm{~dB}$ starting from $100 \mathrm{~mA}$ with Peak 2 is nearly reached to high peak power of $0 \mathrm{dBm}$. Meanwhile, Peak 1 is only $-70 \mathrm{dBm}$ even though the SOA current is increased until $600 \mathrm{~mA}$, as proper adjustment of the polarization state is needed to improve its power. The peak power difference is the highest for BOA followed by NLSOA, as polarization sensitivity of BOA is higher as compared to NLSOA. 


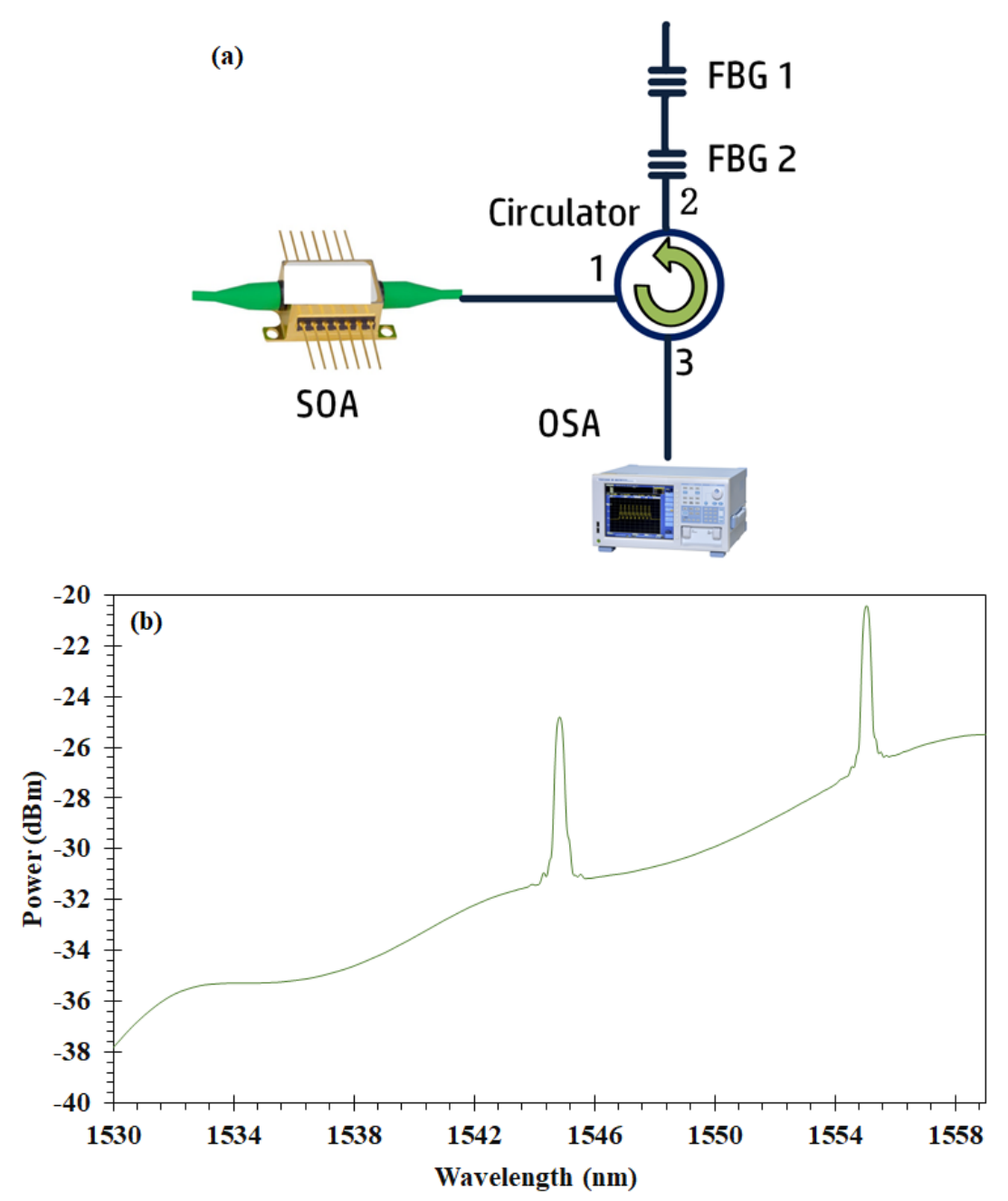

(c)
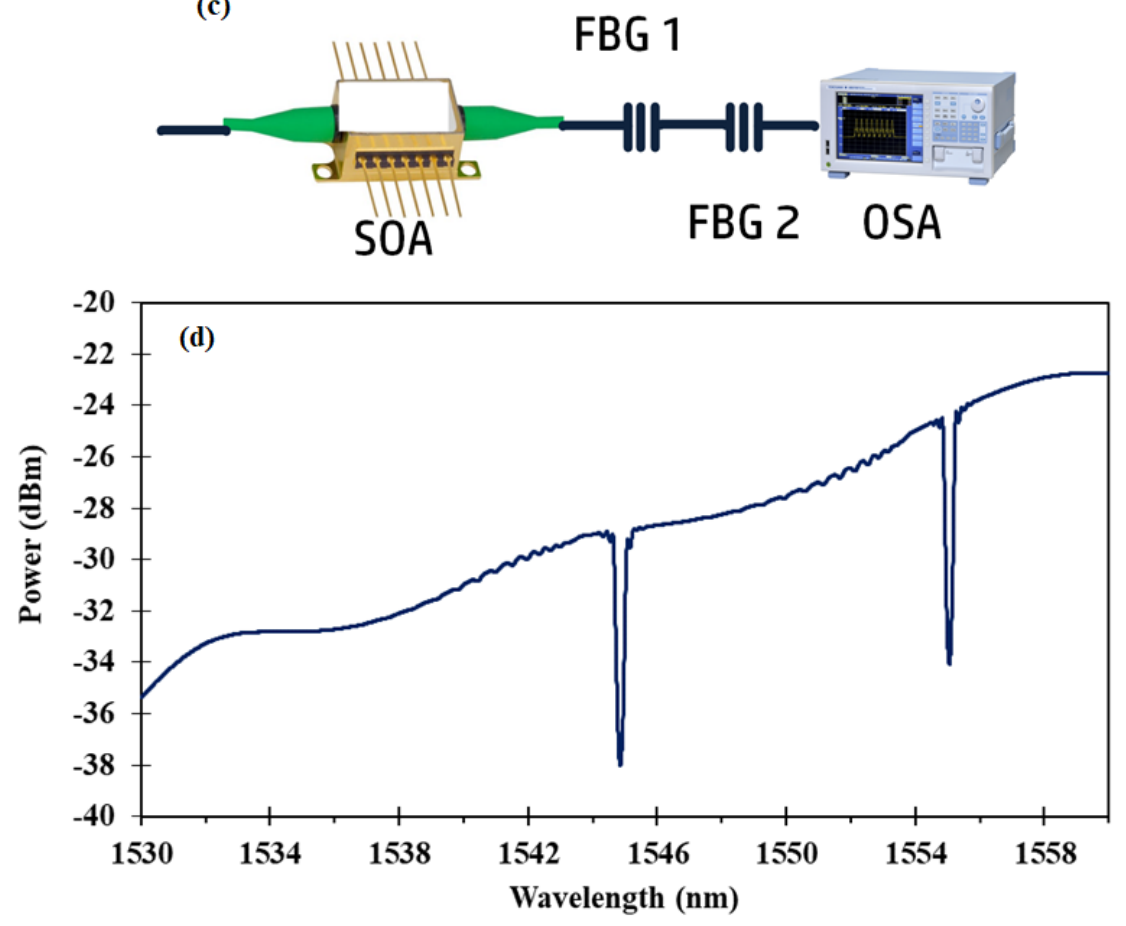

Fig. 4 - (a) The configuration setup to acquire the reflection spectrum of the FBGs; (b) The reflection spectrum of the FBGs at a wavelength of $1545 \mathrm{~nm}$ and $1555 \mathrm{~nm}$; (c) The configuration setup to acquire the transmission spectrum of the FBGs; (d) The transmission spectrum for the FBG at a wavelength of $1545 \mathrm{~nm}$ and $1555 \mathrm{~nm}$ 

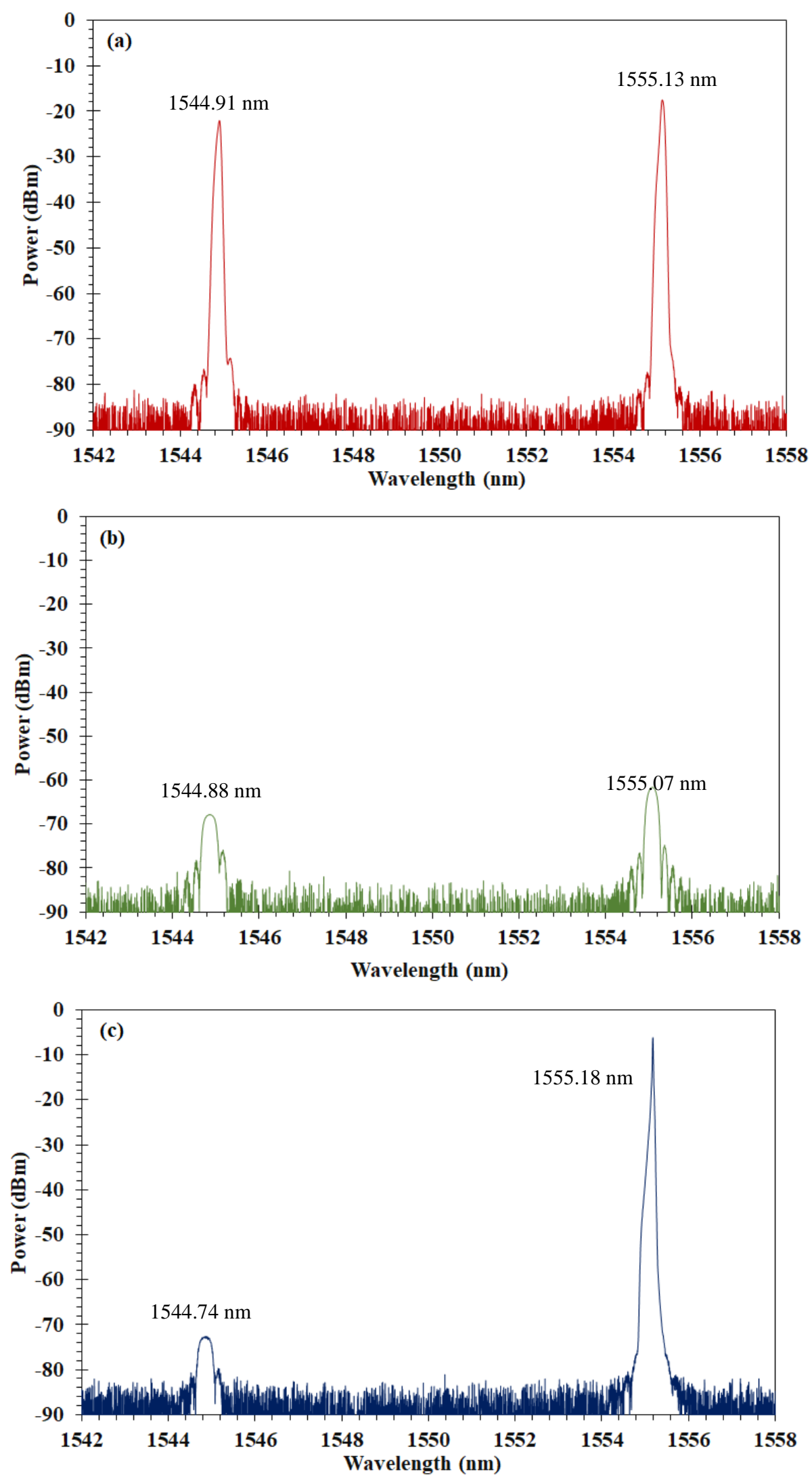

Fig. 5 - The spectrum of DWFL at the same current setting of $200 \mathrm{~mA}$ at different SOA of (a) LSOA; (b) NLSOA; (c) BOA 

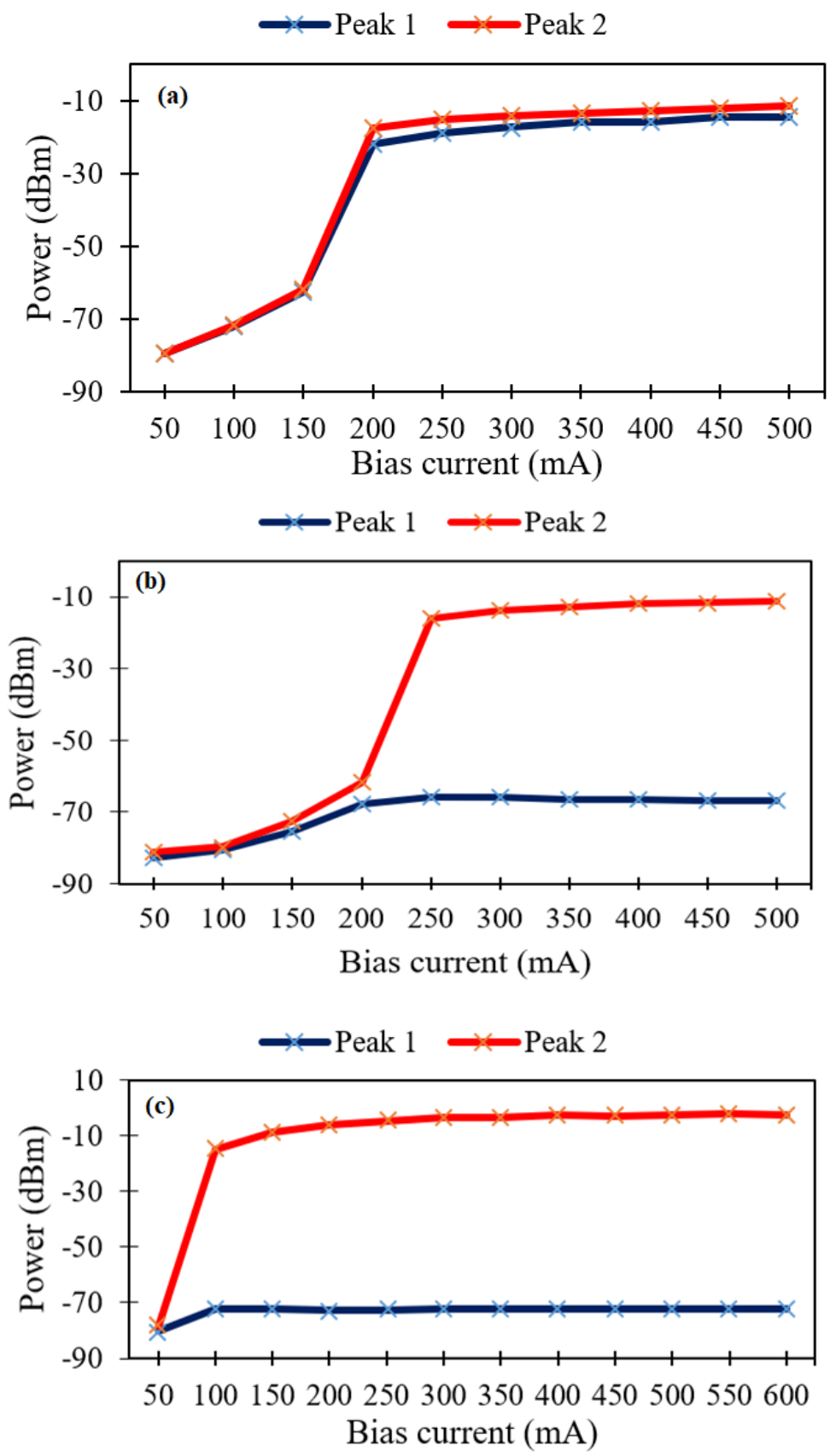

Fig. 6 - The peak power of the DWFL based on (a) LSOA; (b) NLSOA; (c) BOA

Table 2 shows the peak power difference and the measured peak power of LSOA, NLSOA and BOA. BOA has the highest peak power difference at $66.4 \mathrm{~dB}$, followed by NLSOA and LSOA at $6.2 \mathrm{~dB}$ and $4.5 \mathrm{~dB}$, respectively. Meanwhile, the peak power is the highest for BOA at $-6 \mathrm{dBm}$ as compared to other LSOA and NLSOA. NLSOA has the lowest peak power of $-62 \mathrm{dBm}$, while $-17.6 \mathrm{dBm}$ and $-6 \mathrm{dBm}$ peak power for LSOA and BOA, respectively.

Table 2 - The peak power difference and the highest peak power of DWFL

\begin{tabular}{ccc}
\hline Type of SOA & Peak power difference $(\mathbf{d B})$ & Peak Power $(\mathbf{d B m})$ \\
\hline LSOA & 4.5 & -17.6 \\
\hline NLSOA & 6.2 & -62 \\
\hline BOA & 66.4 & -6 \\
\hline
\end{tabular}




\section{Conclusion}

We have demonstrated a DWFL based on different types of SOA utilizing two FBGs as the comb filter. The flattest dual-wavelength is LSOA with $4.5 \mathrm{~dB}$ power difference, followed by NLSOA and BOA with $6.2 \mathrm{~dB}$ and 66.4 $\mathrm{dB}$ power difference, respectively. The flatness of DWFL is degraded with higher polarization sensitivity, according to the value of peak power difference. In our observation, LSOA produces the best lasing performance as its peak power difference is the lowest with high peak power at $-17.6 \mathrm{dBm}$. Even though BOA has the highest peak power at $-6 \mathrm{dBm}$, its flatness is the worst at 66.4 due to high polarization sensitivity as compared to LSOA and NLSOA.ac

\section{Acknowledgement}

This research was financially supported by Universiti Teknologi Malaysia under Research University Grant (Vot. No: 15H37) and by the Ministry of Higher Education under Fundamental Research Grant Scheme (FRGS/1/2017/TK04/UTM/02/2

\section{References}

[1] Sulaiman, A. H., Abdullah, F., Ismail, A., Jamaludin, M. Z., Yusoff, N. Md., \& Mahdi, M. A. (2018). Investigation of multiwavelength laser performance based on temperature variation of PMF and different SOAs. Int. J. Integr. Eng., 10, 244-252

[2] Sulaiman, A. H., Zamzuri, A. K., Yusoff, N. Md., Cholan, N. A., Abdullah, F., Abas, A. F., Alresheedi, M. T., \& Mahdi, M. A. (2018). Broad bandwidth SOA-based multiwavelength laser incorporating a bidirectional Lyot filter. Chinese. Opt. Lett., 16, 1-6

[3] Sun. G., Zhou, Y., Cui, L., \& Chung, Y. (2011). Tunable multiwavelength SOA-fiber ring laser based on Sagnac loop mirror incorporating few-mode high birefringence fiber. Laser Phys., 21, 1899-1902

[4] Sun, B., Hu, K., Chen, D., Wei, Y., Gao, S., \& He, S. (2012). Wavelength-spacing-tunable double-pumped multiwavelength optical parametric oscillator based on a Mach-Zehnder interferometer. J. Light. Technol., 30, 1937-1942

[5] Ahmad, H., Sulaiman, A. H., Shahi, S., \& Harun, S. W. (2009). SOA-based multiwavelength laser using fiber Bragg gratings, Laser Phys., 19, 1002-1005

[6] Xia, L., Shum, P., Wang, Y., \& Cheng, T. H. (2006). Stable triple-wavelength fiber ring laser with ultranarrow wavelength spacing using a triple-transmission-band fiber Bragg grating filter. IEEE Photonics Technol. Lett., $18,2162-2164$

[7] Nguyen, L. V., Hwang, D., Moon, D. S., \& Chung, Y. (2009). Simultaneous measurement of temperature and strain using a Lyot fiber filter incorporated with a fiber Bragg grating in a linear configuration. Meas. Sci. Technol., 20, 1-5

[8] Zhao, X., Dong, M., Zhang, Y., Luo, F., \& Zhu, L. (2019). Switchable multiwavelength and tunable wavelength spacing erbium-doped fiber laser based on a phase-shifted fiber Bragg grating combined with a Mach-Zehnder interferometer. Opt. Laser Technol., 112, 500-507

[9] Feng, T., Jiang, M., Ren, Y., Wang, M., Yan, F., Suo, Y., \& Yao, X. S. (2019). High stability multiwavelength random erbium-doped fiber laser with a reflecting-filter of six-superimposed fiber-Bragg-gratings. OSA Contin. 2, 2526-2538

[10] Ong, Y. S., Kharraz, O. M., Sulaiman, A. H., Abdullah, F., \& Yusoff, N. Md. (2018). Characterization of wideband semiconductor optical amplifiers based on OptiSystem and MATLAB. Int. J. Integr. Eng., 10, 263272.

[11] Sulaiman, A. H., Zamzuri, A. K., Hitam, S., Abas, A. F., \& Mahdi M. A. (2013). Flatness investigation of multiwavelength SOA fiber laser based on intensity-dependent transmission mechanism. Opt. Commun., 291, 264-268

[12] Sulaiman, A. H., Abu Bakar, M. H., Zamzuri, A. K., Hitam, S., Abas, A. F., \& Mahdi M. A. (2013). Investigation of multiwavelength performance utilizing an advanced mechanism of bidirectional Lyot filter, IEEE Photonics J., 5, 1-9

[13] Sulaiman, A. H., Yusoff, N. Md., Cholan, N. A., \& Mahdi M. A. (2018) Multiwavelength fiber laser based on bidirectional Lyot filter in conjunction with intensity dependent loss mechanism, Indones. J. Electr. Eng. Comput. Sci., 10, 401-408

[14] Liu, S., Zhang, Z., Shen, J., \& Yu, K. (2019). Wavelength-spacing adjustable dual-wavelength dissipative soliton resonance thulium-doped fiber laser. IEEE Photonics J., 11, 1-9

[15] Yan, Z., Mou, C., Zhang, Z., Wang, X., Li, J., Zhou, K., \& Zhang, L. (2014). Single polarization, dual wavelength fiber laser based on a 3-stage all fiber Lyot filter. IEEE Photonics Technol. Lett., 26, 1085-1088

[16] Mao, X., Yan, F., Wang, L., Feng S., Peng J., \& Jian, S. (2009). Incorporate, switchable dual-wavelength fiber laser with Bragg gratings written in a polarization-maintaining erbium-doped fiber. Opt. Commun., 282, 93-96.

[17] Sova, R., \& Kim, C., (2002). Tunable dual-wavelength all-PM fiber ring laser. IEEE Photonics Technol. Lett., $14,287-289$ 
[18] Ummy, M. A., Madamopoulos, N., Razani, M., Hossain, A., \& Dorsinville, R. (2012). Switchable dualwavelength SOA-based fiber laser with continuous tunability over the C-band at room-temperature. Opt. Express, 20, 23367-23373

[19] Zhu, T., Zhang, B., Shi, L., Huang, S., Deng, M., Liu, J., \& Li, X., (2016). Tunable dual-wavelength fiber laser with ultra-narrow linewidth based on Rayleigh backscattering. 2016 Conf. Lasers Electro-Optics, CLEO 2016, 24, 1292-1294

[20] Said, Y., \& Rezig, H. (2011). SOAs nonlinearities and their applications for next generation of optical network. Advances in optical amplifiers, Intech, 27-52 